\title{
WIMAX PERFORMANCE IMPACTS IN MULTI-CELL DEPLOYMENTS
}

This paper presents the application of the multiple antenna schemes beamforming (BF) and Multiple Input Multiple Output (MIMO) in the broadband wireless access system WiMAX. The evaluation considers the competing performance demands on link robustness and peak data rate. Configurations for both uplink and downlink are discussed. We conclude that future wireless communications will rely on diverse and adaptive multiple antenna schemes to meet their requirements, in particular in the presence of strong co-channel interference.

\section{Introduction}

The current standard IEEE 802.16e [1], base of the well-known WiMAX Mobile System Profile [2], provides Orthogonal Frequency Division Multiple Access (OFDMA) allowing efficient resource sharing and coping with multipath propagation in dense urban environments. OFDMA is also a dominant candidate for future $4 \mathrm{G}$ wireless communication systems. However, in cellular networks without explicit precaution co-channel interference causes severe performance degradation in terms of coverage and data throughput, particularly in systems with low frequency reuse factor.

WiMAX allows for the multiple antenna solutions beamforming and MIMO which can be exploited to improve radio links. Both approaches utilize more than one antenna element at the base station (BS) for both uplink and downlink, whereas the mobile terminal (MS) is equipped with two receive and one transmit antenna.

Beamforming is mainly used to increase the link budget and to coordinate or even to suppress interference. The basic principle of beamforming is a well-defined phase relation between the individual antenna signals, also referred to as complex beamforming weights.

The WiMAX profile includes MIMO modes with different objectives. In downlink $2 \times 1$ or $2 \times 2$ Space Time Transmit Diversity (STTD) intends to improve the link robustness by the use of Space Time Block Coding (STBC) according to the Alamouti principle. $2 \times 2$ Spatial Multiplexing $(\mathrm{SM})$ is the alternative downlink operation mode. Hereby one user can be served by two independent data streams, thus boosting the overall data rate.

This article gives an overview of the state of the art of multiple antenna solutions. Firstly, in Section II the downlink transmission system is described. After the discussion of the most important functional blocks of a single radio link, the model of the cellular systems with multiple users is presented. Section III covers a detailed performance evaluation on link level and on system level. The relative coverage and throughput improvements depending on transmission mode, antenna configuration and channel model will be given for some exemplary scenarios. Section IV describes two promising algorithms for receive beamforming in uplink before in Section $\mathrm{V}$ the interference suppression capability for different specific scenarios is analyzed by link level simulations. Finally, Section VI summarizes the most important results.

\section{Downlink system model}

In an OFDMA system, the subcarriers of each OFDM symbol are shared among several independent radio links. A certain number of subcarriers are summarized to one subchannel which is the smallest possible user allocation unit in frequency direction. The subcarriers belonging to one subchannel are either physically adjacent or distributed throughout the entire frequency band. In a WiMAX system, the first case is called Adaptive Modulation and Coding (AMC). It is designed for the application of frequency selective scheduling. The latter case is referred to as Partial Usage of Subchannels (PUSC, applicable both in uplink and downlink) and Full Usage of Subchannels (FUSC, downlink only). Both have the capability to exploit frequency diversity in frequency selective channels, i.e. in urban environments with significant delay spread. The WiMAX frame is structured in so called zones. Each zone consists of several OFDM symbols and is based either on AMC, PUSC or on FUSC.

In the following, we will describe the downlink system model in more detail.

\section{A. Link level description}

We now consider the radio link between BS and mobile terminal for one single user, i.e. the signal consists of a certain set of subcarriers in PUSC mode. Moreover, we assume a $2 \times 2$ MIMO

\footnotetext{
* Yejian Chen, Hardy Halbauer, Stephan Saur, Frank Schaich

Alcatel-Lucent Bell Labs, Lorenzstraße 10, D-70567 Stuttgart, Germany, E-mail: Yejian.Chen@alcatel-lucent.de
} 
configuration. Fig. 1 shows a simplified block diagram of the transmitter.

The bits to be transmitted get FEC encoded. After QAM mapping either STBC coding or SM may be applied. Then the symbols are assigned to the used subcarriers of the OFDM symbols within the WiMAX frame. Finally, with the help of an IFFT the transmit signals are generated, and the cyclic prefix is added.

After transmission over the mobile radio channel, the receiver tries to estimate the transmitted bits from the received signals. The principle block diagram of the receiver is shown in Fig. 2. After transformation of the received signals with a FFT, the received data burst is collected. Diversity decoding is applied if STBC is active. Alternatively the data streams are separated if spatial multiplexing is used at the transmitter. After equalization and demodulation, FEC decoding is done. transmit antenna number. $n_{1}$ and $n_{2}$ are independent Gaussian distributed complex noise samples with zero mean and variance $\sigma^{2} / 2$ per dimension, where the index reflects the OFDM symbol, * denotes complex conjugation. A constant channel during the interval of two consecutive OFDM symbols and ideal channel knowledge at the receiver is assumed. A total power constraint is accounted for.

\section{System level description}

From the beginning, it was the intention of the IEEE 802.16e/ WiMAX standard to enable multi-cell deployments including handover capabilities and frequency reuse within the network. The standard is inherently designed to support BSs with three sectors per site, covering 120 degrees each. This is also the configuration we analyze throughout this paper. With this deployment type, a frequency reuse factor of 3 (RU3) where each sector operates in a different frequency band is a natural approach.

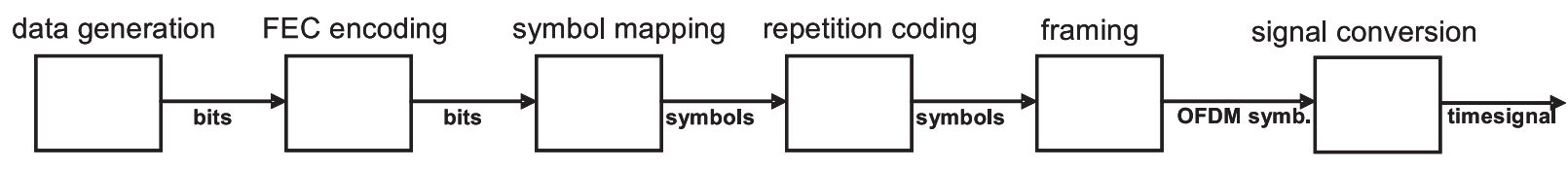

Fig. 1: Block diagram of the transmitter

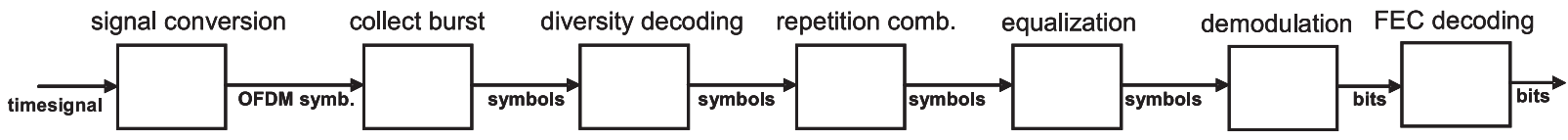

Fig. 2 Block diagram of the receiver

\section{B. Downlink MIMO in WiMAX}

A plethora of multi antenna schemes for downlink are defined in IEEE 802.16e [1]. To reduce complexity both in the BS and in the mobile terminal, the WiMAX Forum has selected two important schemes in its Mobile System Profile [2]. $2 \times 1$ and $2 \times 2$ STBC allow for increasing link robustness. The algorithm is based on the well-known Alamouti principle [3] and will be described roughly in the following. For SM performance studies and algorithm proposals, the interested reader is referred to $[4,5]$.

Let $s_{1}$ and $s_{2}$ be two complex QAM symbols to be transmitted in consecutive OFDM symbols on a single subcarrier. They are fed into the STBC encoder, which delivers four complex symbols at its output, one per transmit antenna and per OFDM symbol. After transmission over the channel, the receiver combines the received symbols to get:

$$
\begin{aligned}
& \tilde{s}_{1}=\left(\left|h_{1}\right|^{2}+\left|h_{2}\right|^{2}\right) \frac{1}{\sqrt{2}} s_{1}+h_{1}^{*} n_{1}+h_{2}^{*} n_{2}=h_{e f f} \frac{1}{\sqrt{2}} s_{1}+n_{l, e f f} \\
& \tilde{s}_{2}=\left(\left|h_{1}\right|^{2}+\left|h_{2}\right|^{2}\right) \frac{1}{\sqrt{2}} s_{2}+h_{2}^{*} n_{1}+h_{1}^{*} n_{2}=h_{e f f} \frac{1}{\sqrt{2}} s_{2}+n_{2, e f f}
\end{aligned}
$$

$h_{1}$ and $h_{2}$ are the complex channel coefficients between the transmit antennas and the receive antenna, where the index reflects the
For the assessment of performance metrics like coverage and data throughput in multi-cell scenarios, the system simulation methodology illustrated in Fig. 3 is used. This analysis includes effects of pathloss, antenna radiation pattern and interference from

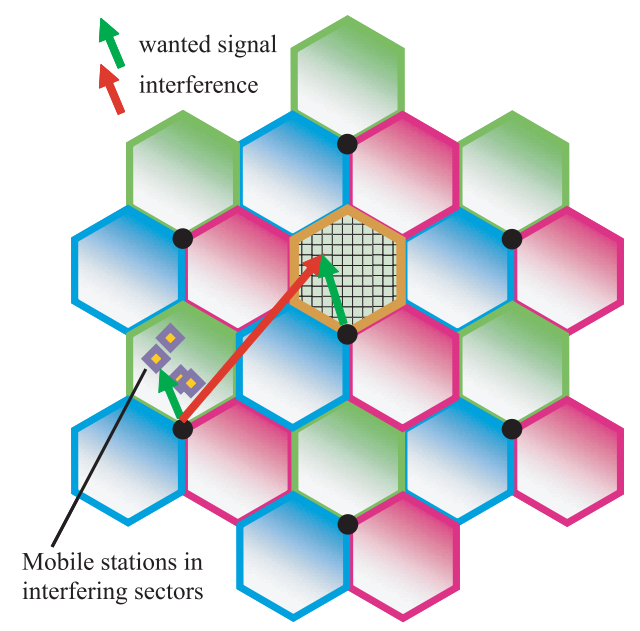

Fig. 3 Considered multi-cell deployment 
neighboring cells assuming a certain frequency reuse factor. Also the impact of downlink beamforming can be investigated.

The deployment consists of 7 BSs and 21 sectors. The different colors indicate the used frequency band, i.e. in Fig. 3 we exemplify a RU3 system. The performance in terms of effective Signal-toInterference-plus-Noise Ratio (SINR) is evaluated at the grid points in the center, green sector, also referred to as reference sector. Mobile terminals causing interference in the reference sector are located randomly in the neighboring sectors. One simulation considers a multitude of these Monte Carlo drops.

As link level simulations give the Packet Error Ratio (PER) for a certain Signal-to-Noise Ratio (SNR) value, the expected data throughput at each grid point and the coverage can be determined easily. The mean sector throughput is the average over the achievable throughput at each grid point of the reference sector. The coverage is the percentage of grid points at which transmission with a data rate above a certain threshold is achieved.

\section{Multi-Antenna Configurations}

Multi-antenna configurations at the BS can significantly reduce the impact of interference in a multi-cell deployment and improve performance. The configurations supported by the WiMAX Mobile System Profile [2] are

\section{- MIMO}

- dynamic beamforming

- combinations of MIMO and beamforming

Concerning interference behavior, the MIMO systems using sector antennas are similar to single antenna systems. In contrast, a beamforming array concentrates the transmitted energy in direction to the addressed user, thus a reduction of the interference in other directions is expected. Combination of MIMO and beamforming promises the benefits of both. Therefore the focus throughout this paper is on the comparative analysis of these configurations.

\section{Downlink performance comparison}

\section{A. Impact of channel correlation on STBC performance}

The channel coefficients $h_{1}$ and $h_{2}$ are time-variant and exhibit deep fades. The gain achievable with STBC is due to the fact that the effective coefficient $h_{\text {eff }}$ from (1) is flattened compared to the original $h_{1}$ and $h_{2}$, i.e. the probability of a deep fade is significantly reduced. Let $\Xi_{1}$ and $\Xi_{2}$ be the events of $h_{1}$ and $h_{2}$ being in deep fade. Then $P_{\text {SISO }}=P\left[\Xi_{1}\right]$ is the probability that the single link in the case of SISO fails. Applying STBC, two links between the transmitter and the receiver are present. The symbol transmitted is lost if both links are in deep fade. Therefore, the joint probability $P_{\text {MISO }}=P\left[\Xi_{1}, \Xi_{2}\right]$ quantifies the probability of losing a symbol during transmission. A simple conversion delivers $P_{M I S O}=P\left[\Xi_{1}\right]$ $P\left[\Xi_{2} \mid \Xi_{1}\right]$. In the case of uncorrelated links, $P\left[\Xi_{2} \mid \Xi_{1}\right]=P\left[\Xi_{1}\right]$ holds. Then we get $P_{M I S O \text {, uncorrelated }}=P\left[\Xi_{1}\right] P\left[\Xi_{2}\right]$. The probability to lose a symbol is thus decreased compared to the SISO case. On the other hand, if the links are fully correlated, we have $P\left[\Xi_{2} \mid \Xi_{1}\right]=1$, i.e. we get $P_{\text {MISO,full uncorrelated }}=P\left[\Xi_{1}\right]$. It follows that the MISO system degrades to SISO performance in the case of full correlation. The probability to lose a symbol due to deep fades in the case of partially correlated links lies in between, i.e.

$$
\begin{aligned}
& P_{M I S O, \text { uncorrelated }}<P_{M I S O, \text { partially uncorrelated }}< \\
& <P_{M I S O, \text { full uncorrelated }} .
\end{aligned}
$$

The following results are obtained with the help of a WiMAX downlink simulator. The data is transmitted in 20 basic allocation units per frame in a PUSC zone. The packet size is 64 Bytes. A tap delay line channel model with six taps is used, each encountering Rayleigh fading, (ITU-R Pedestrian B with $3 \mathrm{~km} / \mathrm{h}$ ). Spatial correlation is included with a correlation matrix per tap [6]. The angular spread is $2^{\circ}$ at the $\mathrm{BS}$ and $35^{\circ}$ at the mobile terminal. The mean angles of departure (AoD) and angles of arrival (AoA) are chosen according to Table 1 .

Angles of departure (AOD) and angles of arrival

Table 1 (AOA) used for simulation

\begin{tabular}{|c|c|c|c|c|c|c|}
\hline & Tap 1 & Tap 2 & Tap 3 & Tap 4 & Tap 5 & Tap 6 \\
\hline AoD & $18.11^{\circ}$ & $24.48^{\circ}$ & $21.11^{\circ}$ & $6.47^{\circ}$ & $23.85^{\circ}$ & $24.24^{\circ}$ \\
\hline AoA & $147.34^{\circ}$ & $50.84^{\circ}$ & $139.08^{\circ}$ & $49.5^{\circ}$ & $260.03^{\circ}$ & $128.93^{\circ}$ \\
\hline
\end{tabular}

Channel estimation is done with the help of pilots and linear interpolation. Convolutional turbo coding with code rate $1 / 2$ is used. Four turbo decoder iterations are carried out.

We compare cases with no correlation, low correlation ( $4 \lambda$ spacing between the antennas at the BS), high correlation $(\lambda / 2$ spacing) and full correlation, where $\lambda$ is the wavelength of the carrier frequency. The results of this study are presented in Fig. 4.

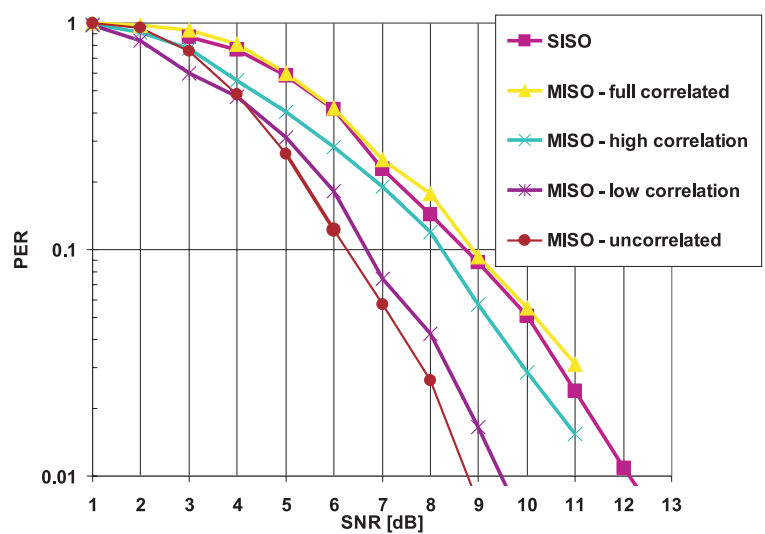

Fig. 4 Impact of correlated links

Obviously, STBC increases the robustness of the link. For all modulation schemes we find a diversity gain of approximately 2.5 $\mathrm{dB}$ of STBC compared to SISO at a PER of $10 \%$. 
Moreover, we have investigated the impact of correlated links.

As expected, STBC with fully correlated links shows the same behavior as SISO. The lower the correlation, the higher is the performance gain. A spacing of $4 \lambda$ can be seen as a typical value for the application of MIMO at the BS. Obviously, we achieve almost the same diversity gain as in the case with uncorrelated links.

\section{B. Interference in multi-cell scenarios}

Since spectrum is a scarce resource, it is desirable to apply better frequency reuse than RU3 up to RU1 so that each sector uses the same frequency band. However, the frequency reuse capability is limited by the interference from neighboring BSs operating in the same frequency band. It is obvious that in this case the interference increases. Especially at the sector borders the SINR can decrease down to $0 \mathrm{~dB}$ or less. In the following text we focus on approaches targeting full RU1 operation enabling the use of the full bandwidth in each sector.

\section{System Configurations and Simulation Setup}

To assess the potential and the limitations of the different approaches and to evaluate the performance impact on throughput and coverage, simulations with the system level simulation tool according to the principles described in section II.C have been performed. The impact of different parameters like frequency reuse factor, PUSC/AMC permutation and antenna configuration is shown by separate examples for each of the impacts. Table 2 shows the antenna configurations, which are taken into account in the following analysis (only partly for each of the examples). The BF arrays have $\lambda / 2$ spacing, the MIMO antennas or MIMO subarrays are separated by $4 \lambda$.

The BS distance depends on the selected pathloss model and is between $750 \mathrm{~m}$ and $1.2 \mathrm{~km}$. The BSs are located in $30 \mathrm{~m}$ height, for the mobile stations $1.5 \mathrm{~m}$ are assumed. The "Spatial Channel Model Extended" (SCME) [7] fading channel profile "urban macro" in combination with the Cost-Hata or SUI [8] pathloss model is applied to the reference signal and the interferers. Also $15 \mathrm{~dB}$ wall penetration loss for indoor coverage and $8 \mathrm{~dB}$ shadow variance is included. The different antenna configurations are modeled with appropriate input parameters and antenna pattern for the SCME model. The total Tx power of $39 \mathrm{dBm}$ is identical for all scenarios. In each interfering sector six users are served, using together all available subchannels. For 300 - 500 Monte Carlo drops of interfering users the total SINR at each location in the reference sector is calculated with the Exponential Effective SINR Mapping (EESM) method [9].

\section{Performance measures}

RU 1 operation is only reasonable if sufficient performance can be achieved. The main performance indicators for the assessment of RU 1 approaches are the area coverage and the mean sector throughput. Whereas the minimum required throughput depends on the services to be deployed, the coverage target is usually in the range of $90-95 \%$ or even higher. The spectral efficiency per sector in bit $/ \mathrm{s} / \mathrm{Hz}$ then is calculated from the total available bandwidth, the mean sector throughput and the complete frame duration.

\section{E. Simulation Results}

In a first example the impact of the frequency reuse factor on the spectral efficiency and the coverage is shown. The antenna configurations BF2-STBC, BF2-SM and BF4-MRC are compared, which correspond to 4-antenna BS and 2-antenna MS and the operation modes MIMO+BF with MIMO receiver at the MS or 4element BF with MRC receiver at the MS. Fig. 5 shows the icdf curves. The curves shifted to the right indicate higher SINR for a larger percentage of the sector area. The PUSC subcarrier allocation with 6 subchannels per interfering user is applied. The dashed lines indicating the RU3 curves are shifted to the right compared to the corresponding RU1 curves, reflecting the higher SINR due to less interference.

In Fig. 6 the corresponding spectral efficiency and the coverage for a minimum data rate of $100 \mathrm{kbit} / \mathrm{s}$ is given. The spectral efficiency is for all RU1 configurations higher than for RU3, but the corresponding coverage is only for the MIMO STBC and for the beamforming configuration above $95 \%$. The RU3 configurations reach almost $100 \%$ coverage with these configurations. This indicates that the MIMO STBC and the BF schemes mainly contribute to the improvement of coverage and link budget. MIMO SM schemes can significantly improve the peak user throughput, but only in the areas where SM can be applied. The MIMO SM coverage is significantly less than the MIMO STBC or BF coverage.

In another exemplary analysis Fig. 7 and Fig. 8 the impact of the permutation mode is analyzed for MIMO+BF and RU1 operation. Both PUSC and AMC mode are applied with random

\begin{tabular}{|c|c|c|c|c|}
\hline \multirow{2}{*}{ Configu-ration } & \multicolumn{2}{|c|}{ Number and type of antenna at } & \multirow{2}{*}{ Transmission mode } & \\
\cline { 2 - 3 } & Base Station (BS) & Mobile Station (MS) & & \multirow{2}{*}{ Single antenna, pure MIMO } \\
\hline SA-SISO & 1 sector antenna & 1 & SISO & \\
\hline SA-STBC & 2 sector antennas & 2 & MIMO STBC & \\
\hline SA-SM & 2 sector antennas & 2 & MIMO STBC & \multirow{2}{*}{ 2-element BF, MIMO+BF } \\
\hline BF2-STBC & 2 two-element arrays & 2 & MIMO SM & \\
\hline BF2-SM & 2 two-element arrays & 2 & MRC & BF \\
\hline BF4-MRC & 1 four-element array & 2 & & \\
\hline
\end{tabular}




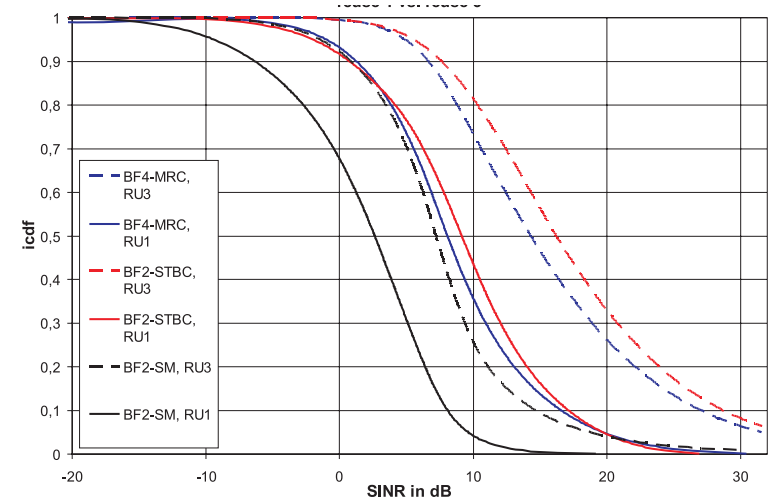

Fig. 5 Comparison of SINR icdf curves of $R U 3$ (dashed lines) and $R U$ 1 (solid lines) for MIMO+BF and BF antenna configurations and PUSC subcarrier allocation

resource allocation to the users. The curves for AMC permutation (solid lines) are shifted to the right part of the diagram, indicating higher throughput at SINR values between 10 and $20 \mathrm{~dB}$. Here

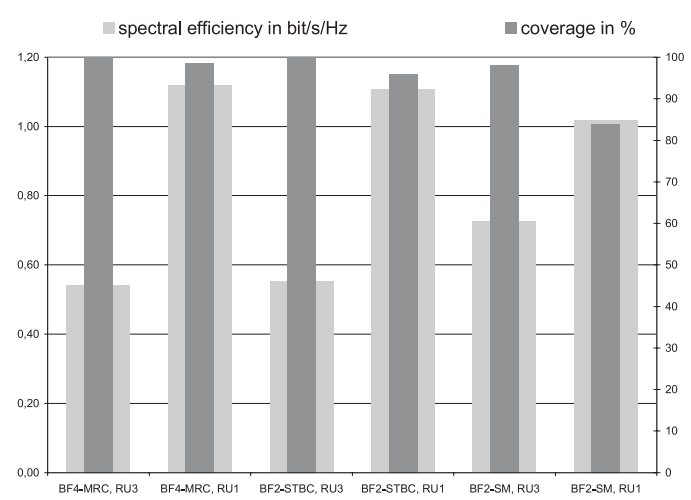

Fig. 6 Spectral efficiency (light grey) and coverage for $100 \mathrm{kbit} / \mathrm{s}$ minimum data rate (dark grey) for $M I M O+B F$ and BF antenna configurations with $R U 3$ and $R U 1$

the higher order modulation schemes can be applied for more points, thus increasing the mean sector throughput. This can be seen also in Fig. 8, where the AMC subcarrier allocation shows for each

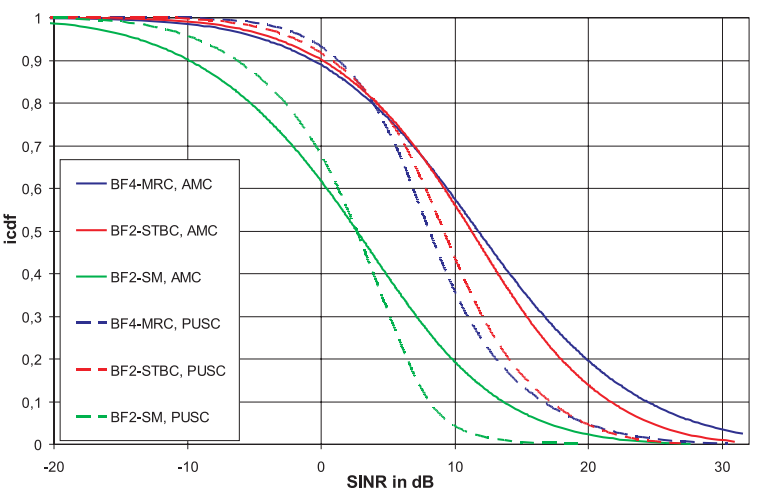

Fig. 7 Comparison of SINR icdf for PUSC and AMC MIMO+BF and BF configurations

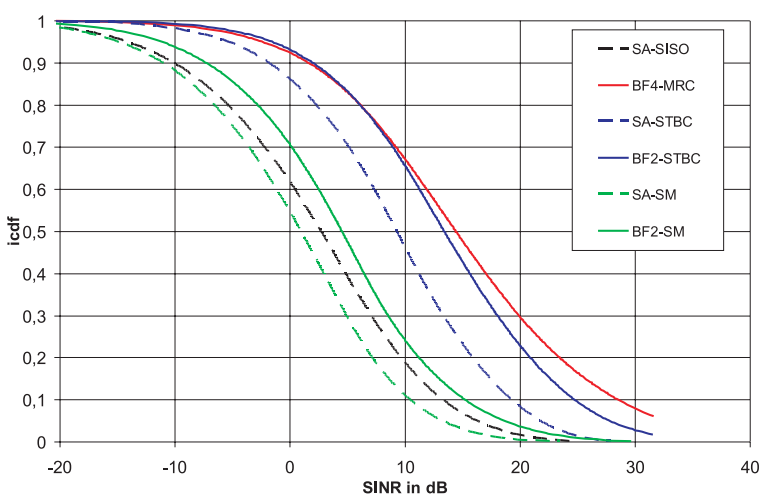

Fig. 9 Comparison of SINR icdf curves for single antenna and multiantenna MIMO and BF configurations

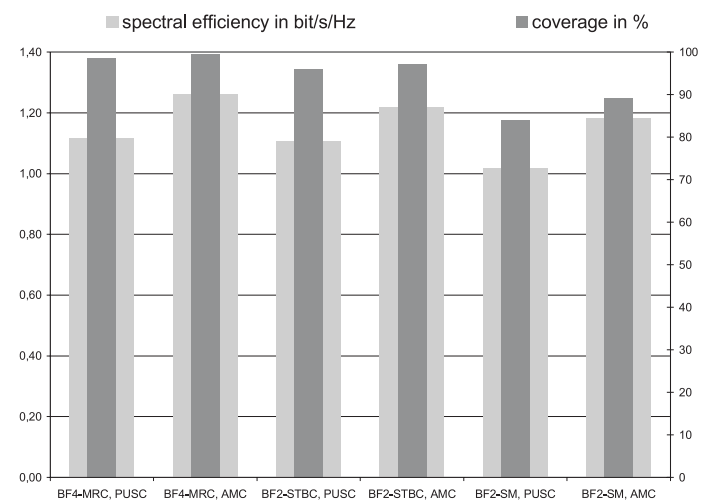

Fig. 8 Spectral efficiency and coverage for PUSC and AMC MIMO+BF and $B F$ configurations

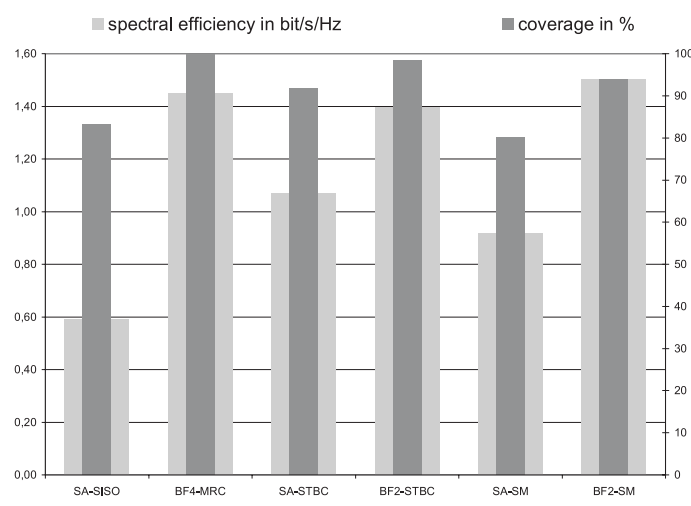

Fig. 10 Comparison of spectral efficiency and coverage for single antenna and multi-antenna MIMO and BF configurations 
antenna configuration an increased spectral efficiency and a slightly increased coverage compared to PUSC.

In a further example Fig. 9 the relation between fixed sector antenna MIMO (dashed lines), MIMO+BF and BF (solid lines) is shown. The results are for AMC in combination with the SUI pathloss model and urban macro profile of the SCME fading channel. In Fig. 9 it can be seen that MIMO+BF with STBC and $\mathrm{BF}$ with 4 elements have almost identical performance, whereas the curve of the fixed sector antenna MIMO is shifted about $5 \mathrm{~dB}$ to the left. MIMO SM with fixed antennas is even worse than SISO, whereas MIMO+BF SM is about $1 \mathrm{~dB}$ better than SISO. The corresponding spectral efficiency and coverage are given in Fig. 10. Whereas the coverage of the fixed antenna configurations is only up to $50 \%-75 \%$, the multi-antenna configurations MIMO+BF STBC and BF reach up to nearly $90 \%$ coverage in this scenario at almost identical spectral efficiency. This clearly indicates that WiMAX systems for RU1 operation with reasonable coverage need to exploit the interference mitigating properties of adaptive multiple antenna solutions, either with BF or with the combination of $\mathrm{MIMO}$ and $\mathrm{BF}$.

\section{Uplink system model}

In the current WiMAX 802.16e standard, the mobile terminal deploys one antenna for uplink signal transmission. The BS has a degree of freedom to utilize diverse antenna configurations with 2 or 4 receive antennas. The beamforming technique is one of the key strategies to combine the useful signal and meanwhile suppress the interference. Throughout this section, the Spatial Maximum Ratio Combining (S-MRC) and the Spatial Minimum Mean Square Error (S-MMSE) beamformers are focused on. Let us firstly consider the narrowband signal model by the system equation,

$$
y=h s+n,
$$

where $s$ is the transmit signal with instantaneous energy per symbol $E_{s}$. The denotations $y, h$ and $n$ stand for complex $N \times 1$ vectors for receive signals with $N$ BS antennas, frequency flat channel coefficients and spatially white Gaussian noise samples with variance $\sigma^{2} / 2$ per dimension. The S-MRC takes the Hermitian of channel estimates as the beamforming weights, i.e.

$$
w_{S-M R C}=\frac{h^{H}}{\|h\|^{2}}
$$

by assuming perfect channel information at the receiver. The operator $\|\cdot\|$ denotes the norm of a vector. The signal combining can be straightforwardly done as

$$
\hat{s}=w_{S-M R C} \cdot y=\frac{h^{H}}{\|h\|^{2}}(h s+n)=s+\frac{h^{H}}{\|h\|^{2}} n .
$$

Hence, the SNR can be computed by

$$
S N R_{S-M R C}=\frac{E\left[s \cdot s^{*}\right]}{E\left[\frac{h^{H}}{\|h\|^{2}} n \cdot\left(\frac{h^{H}}{\|h\|^{2}} n\right)^{*}\right]}=\frac{E_{s}}{E\left[\frac{h^{H}\left(\sigma^{2} I\right) h}{\|h\|^{4}}\right]}=\frac{E_{s}}{\sigma^{2} E\left[\frac{h^{H} h}{\|h\|^{4}}\right]}=E\left[\|h\|^{2}\right] \frac{E_{s}}{\sigma^{2}} .
$$

In the case that co-channel interference is present, the system equation (3) becomes

$$
y=h s+\sum_{i=1}^{M-1} h_{i} s_{i}+n,
$$

where $s_{i}$ is the signal from the $i^{t h}$ interferer, and $h_{i}$ denotes the corresponding channel vector. The number of interferers is given by $M-1$. With the ideal S-MRC beamforming weights in (4), the SINR is consequently

$$
\operatorname{SINR}_{S-M R C}=E\left[\|h\|^{2}\right] \frac{E_{s}}{\sum_{i=1}^{M-1} E\left[\frac{\left\|h^{H} h_{i}\right\|^{2}}{\|h\|^{2}}\right] E_{s_{i}}+\sigma^{2}} .
$$

In (8), it is shown that the S-MRC beamformer does not consider the interference when calculating the weights $w_{S-M R C}$. On the other hand, if the channels $h$ and $h_{i}$ are spatially independent and identical distributed random variables, the S-MRC beamformer yields the array gain $E\left[\|h\|^{2}\right]$ with diversity order $N$. Nevertheless, the prerequisite above is sometimes hardly to be satisfied, especially with $\lambda / 2$ spacing. The S-MRC thus suffers from the interference to a certain degree.

It is well known that the MMSE criterion is linearly optimal in a statistical sense [10]. To investigate the S-MMSE beamformer, equation (7) has to be reformulated in a matrix expression as

$$
y=H s+n,
$$

where $H$ is an $N \times M$ channel matrix, and it holds $H=\left[\begin{array}{lll}h & h_{1} & h_{2}\end{array}\right.$ ... $\left.h_{M-1}\right]$. The signal $s$ is a $M \times 1$ vector, and it holds $s=\left[\begin{array}{lll}s & s_{1} & s_{2}\end{array}\right.$ ... $\left.s_{M-1}\right]^{T}$. Without loss of generality, both the user of interest and the interferers are assumed to have the same transmit signal energy per symbol $E_{s}$. The S-MMSE beamforming weights can be presented in a general form as

$$
w_{S-M M S E}=E_{s} h^{H}\left(E\left[y y^{H}\right]\right)^{-1}=E_{s} h^{H} R_{y y}^{-1},
$$

with the covariance matrix $R_{y y}$ of the received signal vector $y$. The SINR with respect to the signal $s$ of the user of interest is according to [11]

$$
\operatorname{SINR}_{S-M M S E}=\frac{\operatorname{det}\left(R_{y y}\right)}{\operatorname{det}\left(R_{y y}-E_{s} h h^{H}\right)}-1 .
$$

In chapter $V$, it will be shown that the S-MMSE beamformer outperforms S-MRC at low SINR. At high SINR, the S-MRC beamformer converges the performance of S-MMSE with diversity order $N$.

In the link level simulator, pilot based channel estimation is applied. The channel estimates for uplink user $m$ is given by 


$$
\hat{h}^{(m)}=\left[\begin{array}{lllll}
\hat{h}^{(1, m)} & \ldots & \hat{h}^{(n, m)} & \ldots & \hat{h}^{(N, m)}
\end{array}\right]^{T} .
$$

In the expression form of $\hat{h}^{(i, j)}, i$ denotes the receive antenna index, and $j$ denotes the uplink user index. The S-MRC weights can be thus straightforwardly obtained with (4) as

$$
w_{S-M R C}^{(m)}=\frac{\left[\hat{h}^{(m)}\right]^{H}}{\left\|\hat{h}^{(m)}\right\|^{2}} .
$$

For S-MMSE beamformer, the covariance matrix is computed on the basis of the pilot grid. It yields

$$
\hat{R}_{y y}=\frac{1}{M} \sum_{m} \hat{h}^{(m)}\left[\hat{h}^{(m)}\right]^{H} .
$$

In order to increase the robustness of the S-MMSE beamformer, for the matrix inversion a diagonal loading factor $D$ is introduced. This methodology in array processing theory [12] is called Loaded Sample Matrix Inverse (LSMI) beamforming. The LSMI weights for uplink user are given by

$$
\left[w_{S M I}{ }^{(m)}\right]^{T}=\left[\hat{h}^{(m)}\right]^{H}\left[\hat{R}_{y y}+D \cdot \operatorname{tr}\left(\hat{R}_{y y}\right) \cdot I\right]^{-1} .
$$

Finally, the corresponding S-MMSE beamforming weights become

$$
w_{S-M M S E}{ }^{(m)}=\frac{w_{S M I}{ }^{(m)}}{\left\|w_{S M I}{ }^{(m)}\right\|}\left\|E\left[\hat{h}^{(m)}\right]\right\| .
$$

\section{Uplink performance comparison}

In this section, the S-MRC and S-MMSE beamformers are investigated and compared with link level simulations. Firstly, the resulting beam patterns for both methods are presented in Fig. 11. We assume that the BS has 4 receive antennas with $\lambda / 2$ spacing between the elements, and both user - and interferer terminal is equipped with one transmit antenna. The spatial separation of user and interferer is $10^{\circ}$

In Fig. 11(b), it can be observed that the S-MRC beamformer is able to combine the antenna signals exactly in the direction of the user of interest, without considering the direction of the inter- ferer. The disadvantage of S-MRC was already mentioned during the discussion of (8). If the radio channels of interferer and user are spatially independent and identical distributed, not so much performance degradation is introduced by the interferer. Nevertheless, as a matter of fact, $10^{\circ}$ spatial separation does not statistically allow enormous independency. Hence, the interference term in the denominator in (8) cannot be neglected, which finally decreases the SINR. In contrast, the MMSE criterion tries to linearly optimize the SINR, which is shown in Fig. 11(a). The S-MMSE beamformer does not place its main beam to the direction of the user of interest. Instead, it rotates the main beam direction such that the interferer is possibly placed in the direction around a null position, and the user of interest is placed in the direction exhibiting satisfactory array gain.

In Fig. 12, the S-MMSE beam patterns are presented for one user of interest and two interferers. The beamformer has in this situation lower degree of freedom compared to the case with only one interferer in Fig. 11. Nevertheless, the MMSE algorithm establishes an optimal beam to possibly suppress both interferers.

Link level simulations for the S-MRC and S-MMSE beamformers were carried out for AMC mode. The simulation parameters are given in Table 3 . The simulations focus on the scenario for one user of interest and one interferer with spatial separations $10^{\circ}$ and $20^{\circ}$. As fading channel model, ITU-R Pedestrian B with 3 km/h was used. Fig. 13 and Fig. 14 show the coded PER and data throughput for the case with $10^{\circ}$ spatial separation between user of interest and interferer. Fig. 13 shows that S-MMSE outperforms its counterpart at low SINR, and S-MRC is able to suppress the interferer to a certain degree at high SINR. The relative beamforming gain of S-MMSE over S-MRC ranges from $0.8 \mathrm{~dB}$ to $2.3 \mathrm{~dB}$ with respect to $10 \%$ PER, depending on the modulation scheme.

Fig. 14 illustrates the throughput benefit from another aspect. By observing the SINR at 10\% PER of the S-MMSE beamformer, e.g. approximately $7.5 \mathrm{~dB}$ for 64QAM, the throughput benefit of S-MMSE over S-MRC is roughly $330 \mathrm{kbps} /$ subchannel. The throughput benefit of the S-MMSE with other modulation schemes can be similarly observed (16QAM: $330 \mathrm{kbps} /$ subchannel; QPSK: 200 kbps/subchannel).

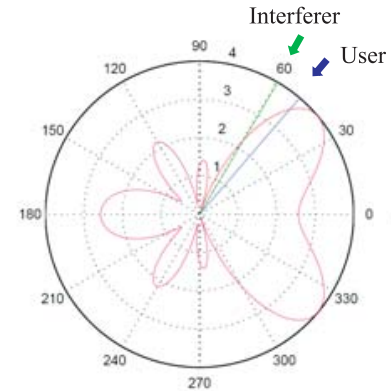

(a)

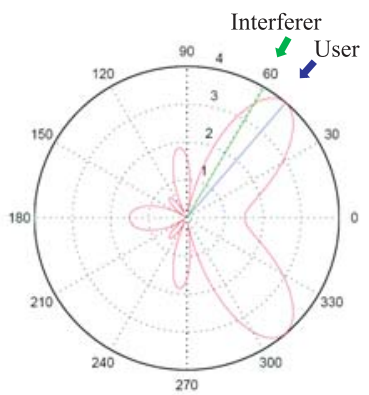

(b)

Fig. 11 S-MMSE (a) and S-MRC (b) beamformer pattern

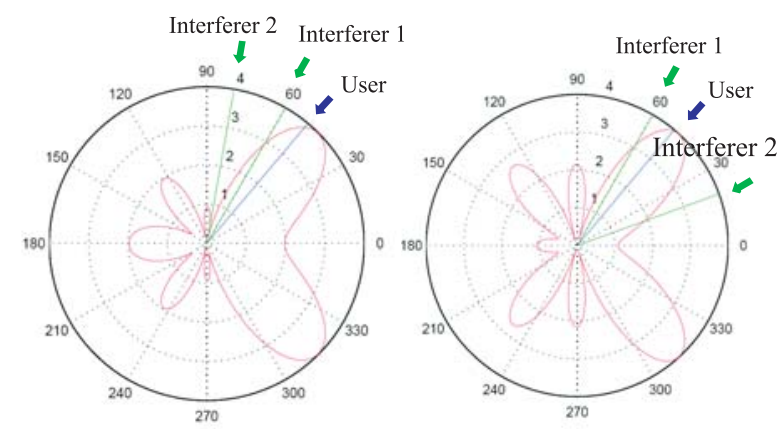

Fig. 12 S-MMSE beam patterns with two interferers 


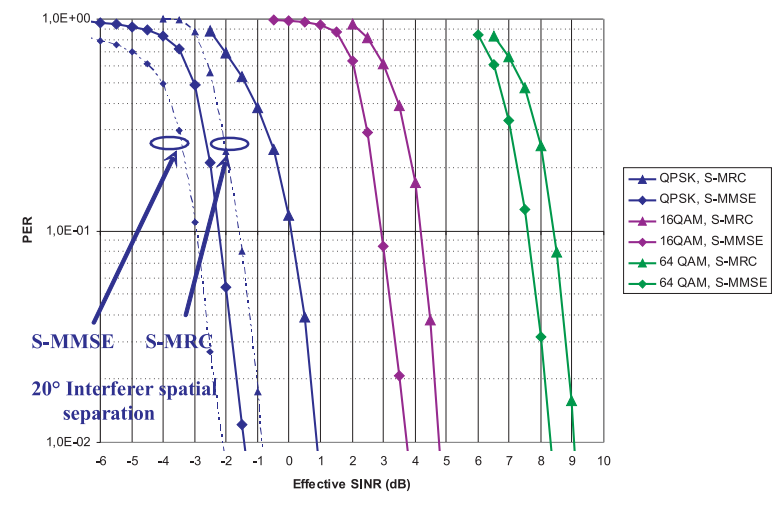

Fig. 13 PER curves with $10^{\circ}$ spatial separation

Simulation parameters

Table 3

\begin{tabular}{|l|l|}
\hline Remarks & Parameters \\
\hline Number of antenna elements & 4 \\
\hline Antenna spacing & $0.5 \lambda$ \\
\hline Beamforming method & S-MMSE and S-MRC \\
\hline FEC coding rate & $1 / 2$ \\
\hline FEC option & Convolutional Turbo Code (CTC) \\
\hline Modulation option & QPSK, 16QAM, 64QAM \\
\hline FFT size & 1024 \\
\hline OFDM permutation mode & AMC 2×3 \\
\hline $\begin{array}{l}\text { Packet size for the single user } \\
\text { inside of one OFDM uplink frame }\end{array}$ & $\begin{array}{l}60 \text { Bytes (QPSK, 16QAM), 54 } \\
\text { Bytes (64QAM) }\end{array}$ \\
\hline Interferer separation spacing & $10^{\circ}$ or 20 \\
\hline $\begin{array}{l}\text { Switch to enable or disable the } \\
\text { sounding }\end{array}$ & Enable \\
\hline Subchannel rotation & OFF \\
\hline Channel & Pedestrian B 3 km/h (PedB3) \\
\hline
\end{tabular}

In Fig. 13 the interference suppression capability is additionally presented for S-MMSE and S-MRC with $20^{\circ}$ interferer spatial separation. The absolute performance is better and the relative performance gap between S-MMSE and S-MRC decreases. The behavior is already indicated in the section IV, that the S-MRC approaches S-MMSE performance due to insignificant spatial correlation between the fading channels of the user and that of the interferer.

\section{Conclusion}

We have discussed various applications of multiple antenna configurations in both uplink and downlink for the mobile broadband wireless access system WiMAX, which is based on the current

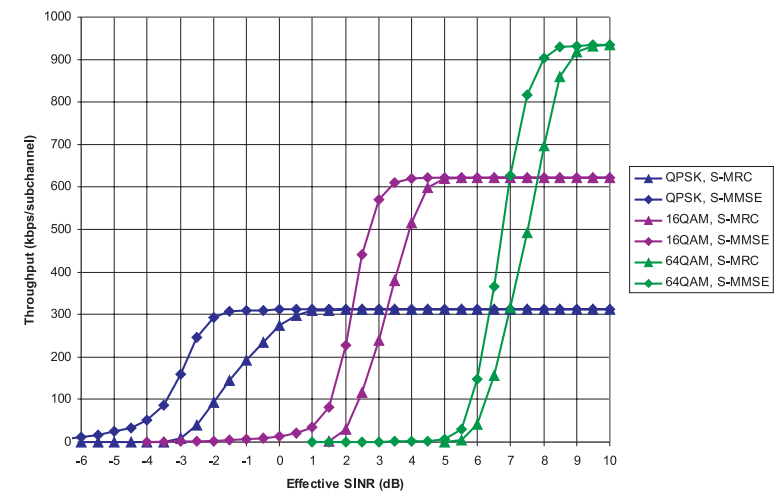

Fig. 14 Throughput curves with $10^{\circ}$ spatial separation

IEEE 802.16e standard. Firstly, it was shown that the exploitation of diversity in partly decorrelated channels improves the link level performance using $2 \times 1$ MISO STBC mode.

However, on system level additional criteria for the correct selection of the appropriate operation mode can be derived. Downlink beamforming as well as MIMO STBC improve the robustness of a link, whereas MIMO SM targets a high spectral efficiency. The latter requires uncorrelated MIMO channels and a high SINR, which limits the application basically in dense urban environments near to the BS. The combination of MIMO and beamforming allows for a further decorrelation of the MIMO channels and thus enlarges the application area of MIMO SM.

Finally two uplink receive beamforming algorithms, namely SMRC and S-MMSE were presented and compared. S-MRC requires uncorrelated channels between user of interest and interferer to exploit a diversity gain. The beam pattern is directed to the user of interest without consideration of the interferers. S-MMSE explicitly optimizes the resulting SINR by selecting the beamforming weights such that the interferers are located closely to nulls of the beam pattern. It was shown that S-MMSE is superior to its counterpart.

Generally, future broadband wireless access systems, e.g. $802.16 \mathrm{~m}$, have to fall back on diverse options for multiple antenna systems to fulfill their manifold performance requirements. Multiple antenna systems can therefore be seen as one basic building block for future wireless communications.

\section{Acknowledgment}

This work has been supported by the German Ministry for Education and Research (BMBF) within the EUREKA MEDEA+ project MIMOWA, which is kindly acknowledged.

\section{References}

[1] IEEE 802.16Rev2/D3: Air Interface for Broadband Wireless Access Systems, February 2008

[2] WiMAX Forum Mobile System Profile Release 1.0, Revision 1.6.1, 2008. 
[3] ALAMOUTI, S. M.: A Simple Transmit Diversity Technique for Wireless Communications, IEEE Journal on Selected Areas in Communications, 8/1998, pp. 1451-1458.

[4] KANG, H.G., PARK, J., AN, T., OH, J., SONG, I.: An ML Decoding Algorithm with Reduced Complexity for Multi-Input MultiOutput Systems, Intern. Symposium on Signals, Systems and Electronics ISSSE '07, 2007, pp. 339-342.

[5] YOO, B., LEE, K. H., LEE, C.: Implementation of IEEE 802.16e MIMO-OFDMA Systems with K-BEST Lattice Decoding Algorithm", Intern. Conference on Consumer Electronics ICCE 2007, Digest of Technical Papers, 2007, pp. 1-2.

[6] IEEE 802.16m Evaluation Methodology Document (EMD), 2008.

[7] 3GPP Technical Report 25.996: Spatial channel model for Multiple Input Multiple Output (MIMO) simulations, V6.1.0, 2003.

[8] ERCEG, V. et al., "Channel Models for Fixed Wireless Applications", IEEE 802.16.3c-01/29r5, June, 2003.

[9] TSAI, S., SOONG, A.: Effective SNR mapping for modelling frame error rates in multiple-state channels, 3GPP2-C30-20030429-010, April 2003.

[10] HAYKIN, S.: Adaptive Filter Theory, Prentice Hall, 1996.

[11] PAULRAJ, A., NABAR, R., GORE, D.: Introduction to Space-Time Wireless Communications, Cambridge, 2003.

[12] VAN TREES, H. L. : Optimum Array Processing, Part IV of Detection, Estimation, and Modulation Theory, John Wiley \& Sons, 2002. 